\title{
INFLUENCE OF CLIMATIC FACTORS ON POPULATION DENISTY OF LAND SNAILS INFESTING SOME CROPS AND THEIR DAMAGE QUESSNA DISTRICT (MINUFYA GOVERNORATE).
}

Asran, A. A. ; T. M. S. Keshta; M.M. Mortada and Fatma K. Khider Plant protection Research Institute, Agric. Res. Center, Dokki, Egypt

\begin{abstract}
This study was conducted under field conditions at Quessna district, Monufya Governorate during two successive seasons 2009 and 2010. The present work aimed to throw light on the effect of climatic factors temperature degree and relative humidity on land snails population and their damage to some stand crops( Clover, Lettuce and Navel orange ).

The obtained results could be summarized as follows:-

1)- Monacha cartusiana (Muller) and Eobania vermiculata (Muller) were only observed infesting Clover, Lettuce and Navel orange during the experimental period. Also, the the ecological studies indicated that $M$. cartusiana was found in abundance on Clover and Lettuce both, meanwhile Navel orange had $E$. vemiculata more than the another, snail.

2) - Clover was the most attractive to $M$. cartusiana followed by Lettuce and Navel orange, whereas, the last crop was the most attractive to E. vermaculata than Clover and Lettuce.

3)- The land snails population was fluctuated during the experimental crop life from month to month due to variations in temperature degree and relative humidity. The results indicate that there is a positive correlation between snail population and the relative humidity, meanwhile it is negative with the temperature degree. The highest population (115.5 individuals) was recorded in February (16 C \&59.9 $\%$ R.H.) and the lowest one (33 individuals) in May (29 C \& 54.7\% R.H.).

4)- The obtained results showed that the highest infestation of land snails $(0.89 \%$, $0.83 \%$ and $0.64 \%)$ and the lowest $(0.45 \%, 0.49 \%$ and $0.19 \%)$ for Clover, Lettuce and Navel orange were recorded in March (18.6C \& 59.7\% R.H.)and in May (29 C \& 54.7\% R.H.), respectively.

$5)$ - The obtained results proved that the most suitable geographical conditions to increase the population of land snails are low temperature degree and height relative humidity. Also, it is added that, there is a positive correlation between population of land snails and their damage to the three tested crops.
\end{abstract}

\section{INTRODUCTION}

The phylum Mollusca is probably the third most important animal group after the arthropods and vertebrates. Snails and slugs belong to the class Gastropoda.

Few species of snails and slugs appear to have been aversely affected by human activities, apart from the localized effects of habitat destruction, while several species have extended their geographical distribution. Snails and slugs have become considerably more abundant as the result of agricultural and horticultural activities. 
Asran, A. A. et al.

The great damage of gastropod to many agricultural and horticultural crops in Egypt has become increasing apparent over the past 30 or 40 years. Data indicated that the damage caused by gastropods are common after the warm wet summer and autumn, although gastropods populations are reduced under dry conditions. However accurate information on the economic importance of gastropods are difficult to be obtained due to its negligence and tends to be more unreliable than that for many important insect pests.

Mollusca are important pest of fruit trees, vegetables, medical plants, ornamental plants and field crops (El-Okda, 1984). The main damage caused by $M$. cartusiana to fresh plant of the Egyptian clover was found to be about $5.65 \%$ (5.082 tons/ feddan ) four consecutive cutting (Okka, 2003).

The aim of the present study is throw light on effect of climatic factors on the population density of some land snails infesting some crops and their damage.

\section{MATERIALS AND METHODS}

Population density of land snails infesting some field crops:

The population density of the land snails was studied some field in crops; Egyptian clover lettuce and navel orange at Begarim, village, Quessina district at Minufiya, Governorate during two successive years 2009-2010.

An area of about one Feddan was selected for each crop. Five replicates (traps) of quadrate sample size $(50 \mathrm{~cm} \times 50 \mathrm{~cm})$ were randomly examined in each chosen area twice per month during the growing season of each crop. Examination was done during early morning before sunrise.

All snails found on either plants or soil surface in the quadrate were counted and left in their initial places (Baker, 1988). Data concerning temperature and relative humidity during the period of study were obtained from metrological station of Minufiya Governorate

\section{Land snails damage estimation:}

Estimation of damage caused by land snails to the Egyptian clover, lettuce and navel orange was conducted at Begarim locality, Quessina district, Minufiya, Governorate. All leaves and stems of plant (for lettuce and clover) or 20 leaves of the nearest branch of the tree (for navel orange trees) were examined in the tested area one per month during the two successive growing seasons 2009 -2010. Temperature degree and relative humidity during the period of study were recorded through out the collecting time. The obtained data were calculated according to the following equation.

Total No. of damaged leaves

Damage $\%=\frac{----------}{\text { Total No. of inspected leaves }} \times 100$

The land snails damage was calculated in other two ways by applying the next formula:

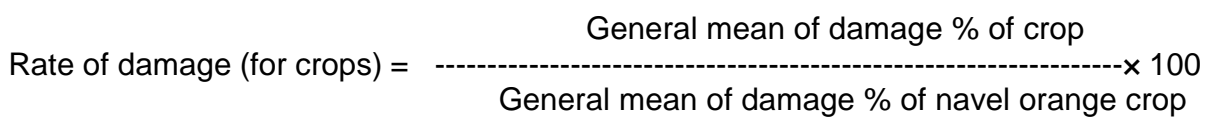


Damage\% of month
Rate of damage (for month) $=\begin{array}{r}--- \\ \text { Damage\% of May }\end{array}$

\section{RESULTS AND DISCUSSION}

This study deal with the land snails population and their damage to different field crops (Clover, Lettuce and Navel orange) under field conditions:

The tabulated data in Table (1) illustrated that Monacha cartusiana (Muller) and Eobania vermiculata (Muller) were only observed on each of clover, lettuce and navel orange during the experimental period. The recorded figures cleared that $M$. cartusina was found in more number on clover and lettuce mean while navel orange trees has E.vermiculata more than $M$. cartusiana. The calculated population density of $M$. cartusina and $E$. vermiculata on the previous mentioned crops for February month was (87\% and $13 \%),(100 \%$ and $0.0 \%)$ and $(33.3 \%$ and $66.7 \%)$ on clover, lettuce and navel orange, respectively. Also, the presented data in Table (2) confirmed with the former results. Besides the presented figures of the rate of collected snails from varied crops were confirmed with the previous results. The studied crops could be arranged in a descending order according to the rate of the collected snails for February month as follow:-

Clover (2.26)> lettuce (1.34)> Navel orange (1). The arrangement of the crops was similar for the rest months. As well as, the data in Table (2) proved that Lettuce $(100 \%)$ was more attractive to M. cartusina followed by Clover (91.3) and Navel orange (33.3\%), meanwhile E. vermaculata was more attractive to Navel orange $(66.7 \%)$ followed by Clover $(13 \%)$ and Lettuce $(0.0 \%)$ for February month. Moreover, the recorded results in Table (1) proved that the population of snails was fluctuated during the experimental crops from month to month because of the variations in each of temperature degree and the relative humidity. The snails population for February month (16Co and $59.9 \%$ R.H.), had the highest mean number of the collected land snails from Clover (115.5 individuals), Lettuce (68.8 individuals) and Navel orange (51 individuals), meanwhile, the lowest mean number was recorded in May month (29 Co and 54.7\% R.H.) 33, 20 and 17 individuals respectively for the same mentioned crops formally. These findings indicate that there is appositive link between snails population and Relative humidity, mean while it is negative with temperature degree. The experimental months and their recorded temperature degree and relative humidity could be arranged discerningly according the rate of snail populations follow:-

February month (16Co \& $59.9 \%$ R.H.) 3.5> January (15.1 Co \&59.9\%R.H.) $2.79>$ March (18.6 Co\&59.7\%R) 2.36> December (19 Co \&59.9\% R.H.) $2.15>$ April (22.3 \&59.7\%R .H.) $1.79>$ May (29 Co \& 54.7\% R.H.) 1 for Clover crop Month arrangement for Navel orange corresponding to rate of snails population took the same trend approximately. 
Asran, A. A. et al. 
Table (2): Host plant preference in relation to land snails population during two successive years 2009-2010.

\begin{tabular}{|c|c|c|c|c|c|c|c|}
\hline \multirow{2}{*}{ Month } & \multirow{2}{*}{ Crop } & \multirow{2}{*}{$\begin{array}{c}\text { Ave.of Total } \\
\text { collected } \\
\text { snails 5/traps/ } \\
3 \text { nights }\end{array}$} & \multirow{2}{*}{$\begin{array}{c}\text { Rate(to } \\
\text { navel } \\
\text { orange) }\end{array}$} & \multicolumn{2}{|c|}{ M. cartusiana } & \multicolumn{2}{|c|}{$\begin{array}{c}E . \\
\text { vermiculata }\end{array}$} \\
\hline & & & & $\begin{array}{l}\text { Av. } \\
\text { No. }\end{array}$ & $\%$ & $\begin{array}{l}\text { Av. } \\
\text { No. }\end{array}$ & $\%$ \\
\hline \multirow{3}{*}{ November } & Clover & 53.5 & 2.15 & 51.5 & 96.26 & 2 & 3.74 \\
\hline & Lettuce & 29.6 & 1.2 & 27.3 & 92.2 & 2.3 & 7.8 \\
\hline & Navel orange & 24.8 & 1 & 7.0 & 28.3 & 17.8 & 71.7 \\
\hline \multirow{3}{*}{ December } & Clover & 71.5 & 2.2 & 66.5 & 93 & 5 & 7 \\
\hline & Lettuce & 35.4 & 1.14 & 35.4 & 100 & 0.0 & 0.0 \\
\hline & Navel orange & 31 & 1 & 10 & 32.3 & 21 & 67.7 \\
\hline \multirow{3}{*}{ January } & Clover & 92 & 2.2 & 81 & 88.5 & 11 & 12 \\
\hline & Lettuce & 58 & 1.4 & 55 & 94.8 & 3 & 5.2 \\
\hline & Navel orange & 42 & 1 & 18 & 42.9 & 24 & 57.1 \\
\hline \multirow{3}{*}{ February } & Clover & 115.5 & 2.26 & 105.5 & 91.3 & 10 & 8.7 \\
\hline & Lettuce & 68.8 & 1.34 & 68.8 & 100 & 0.0 & 0.0 \\
\hline & Navel orange & 51 & 1 & 17 & 33.3 & 34 & 66.7 \\
\hline \multirow{3}{*}{ March } & Clover & 78.2 & 2.3 & 70.2 & 89.8 & 8 & 10.2 \\
\hline & Lettuce & 37.6 & 1.1 & 34.6 & 92 & 3.0 & 8.0 \\
\hline & Navel orange & 34 & 1 & 12 & 35.3 & 22 & 64.7 \\
\hline \multirow{3}{*}{ April } & Clover & 58.5 & 2.9 & 52 & 88.8 & 6.5 & 11.2 \\
\hline & Lettuce & 28.8 & 1.4 & 24 & 83.3 & 4.8 & 16.7 \\
\hline & Navel orange & 20.4 & 1 & 7 & 34.3 & 13.4 & 65.7 \\
\hline \multirow{3}{*}{ May } & Clover & 33 & 194 & 32 & 97 & 1 & 3 \\
\hline & Lettuce & 20 & 1.17 & 19 & 95.0 & 1.0 & 5 \\
\hline & Navel orange & 17 & 1 & 8 & 47 & 9 & 53 \\
\hline
\end{tabular}

Ismail (1997) and El-Deeb et al, (1999) indicated that the highest population density values of $M$. cartusiana were found during spring months as compared with other ones. Also, Abdel-Aal (2001) reported that the population density of M.cartusiana increased during spring Month. Sebay et al., (2009) recorded that the population density of E. vermaculata on Navel orange fluctuated on soil surface and tree trunks during the year months.

The presented data in Table (3) show the land snails damage percentages to Clover, Lettuce and Navel orange and its relation to each of land snails population and the dominant geographical conditions (Temperature and Relative Humidity ). The obtained figures (table 3) cleared that the highest damage was recorded in March (18.6 Co \&29.7\% R. H.), $0.89 \%, 0.83 \% \%$ and $0.64 \%$ for Clover, Lettuce and Navel orange, respectively. Meanwhile, the lowest damage observed in May (29 Co \&54.7\% R. H.), $0.45 \%, 0.49 \%$ and $19 \%$ for the same crops arrangement previously. The tabulated data in Table(3) also added that the rate of damage of the tested crops during month related to May proved that the tested crops had survey damage during March ( in rate 1.98). The rates of the other months ranged between them because of increasing land snails population.

The experimental month could be arranged them in descending order according to the rate of land snail damage as follows :- March $1.98>$ April $1.9>$ February $1.7>$ December $1.38>$ January $1.27>$ May $1.0>$ 
Asran, A. A. et al.

November 0.76 for Clover crop. The arrangement of the rest tested crops was similar.

Table (3): Damage percentage for different crops caused by Monacha cartusiana and Eobania vermiculata land snails At Quessna district; Minufya Governorate during two successive years 2009-2010

\begin{tabular}{|c|c|c|c|c|c|c|c|c|c|}
\hline \multirow[b]{2}{*}{ Month } & \multirow[b]{2}{*}{ Year } & \multicolumn{2}{|c|}{ Average of } & \multicolumn{2}{|c|}{ Clover } & \multicolumn{2}{|c|}{ Lettuce } & \multicolumn{2}{|c|}{ Navel orange } \\
\hline & & $\begin{array}{c}\text { Temp. } \\
\text { d C }\end{array}$ & R.H\% & $\underset{\%}{\text { Damage }}$ & Rate & $\underset{\%}{\text { Damage }}$ & Rate & $\underset{\%}{\text { Damage }}$ & $\begin{array}{c}\text { Monthly } \\
\text { Rate(to } \\
\text { May) }\end{array}$ \\
\hline \multirow[t]{3}{*}{ November } & 2009 & 21.4 & 62.3 & 0.22 & \multirow{3}{*}{0.76} & 0.16 & \multirow{3}{*}{0.86} & 0.14 & \multirow{3}{*}{0.84} \\
\hline & 2010 & 16.5 & 57.5 & 0.45 & & 0.21 & & 0.18 & \\
\hline & mean & 19 & 59.9 & 0.34 & & 0.19 & & 0.16 & \\
\hline \multirow[t]{3}{*}{ December } & 2009 & 16.1 & 65.7 & 0.42 & \multirow{3}{*}{1.38} & 0.25 & \multirow{3}{*}{0.63} & 0.20 & \multirow{3}{*}{1.1} \\
\hline & 2010 & 14.8 & 63.7 & 0.82 & & 0.36 & & 0.22 & \\
\hline & mean & 15.5 & 64.7 & 0.62 & & 0.31 & & 0.21 & \\
\hline \multirow[t]{3}{*}{ January } & 2009 & 15.9 & 63.7 & 0.41 & \multirow{3}{*}{1.27} & 0.33 & \multirow{3}{*}{0.8} & 0.12 & \multirow{3}{*}{1.89} \\
\hline & 2010 & 14.3 & 55.4 & 0.73 & & 0.44 & & 0.18 & \\
\hline & mean & 15.1 & 59.6 & 0.57 & & 0.39 & & 0.15 & \\
\hline \multirow[t]{3}{*}{ Febraury } & 2009 & 15.8 & 66.3 & 0.83 & \multirow{3}{*}{1.71} & 0.78 & \multirow{3}{*}{1.53} & 0.29 & \multirow{3}{*}{0.8} \\
\hline & 2010 & 16.2 & 53.4 & 0.71 & & 0.72 & & 0.43 & \\
\hline & mean & 16 & 59.9 & 0.77 & & 0.75 & & 0.36 & \\
\hline \multirow[t]{3}{*}{ March } & 2009 & 18.2 & 65.9 & 0.95 & \multirow{3}{*}{1.98} & 0.86 & \multirow{3}{*}{1.69} & 0.72 & \multirow{3}{*}{3.37} \\
\hline & 2010 & 19 & 53.4 & 0.82 & & 0.79 & & 0.56 & \\
\hline & mean & 18.6 & 59.7 & 0.89 & & 0.83 & & 0.64 & \\
\hline \multirow[t]{3}{*}{ April } & 2009 & 21.4 & 59.2 & 0.80 & \multirow{3}{*}{1.9} & 0.58 & \multirow{3}{*}{1.63} & 0.70 & \multirow{3}{*}{3.62} \\
\hline & 2010 & 23.1 & 51.0 & 0.89 & & 0.91 & & 0.53 & \\
\hline & mean & 22.3 & 55.1 & 0.85 & & 0.75 & & 0.62 & \\
\hline \multirow[t]{3}{*}{ May } & 2009 & 31.7 & 56.0 & 0.56 & \multirow{3}{*}{1.0} & 0.50 & & 0.21 & \\
\hline & 2010 & 26.2 & 53.4 & 0.33 & & 0.47 & 1.0 & 0.16 & 1.0 \\
\hline & mean & 29.0 & 54.7 & 0.45 & & 0.49 & & 0.19 & \\
\hline $\begin{array}{l}\text { General } \\
\text { mean }\end{array}$ & & & & 0.64 & & 0.53 & & 0.33 & \\
\hline $\begin{array}{l}\text { Crop rate to } \\
\text { navel } \\
\text { orange }\end{array}$ & & & & 1.9 & & 1.6 & & 1.0 & \\
\hline
\end{tabular}

This results agree with Ismail (2004), and Ghada Refaat (2004) mentioned that the percentages of damage were increased or decreased according to temperature and air humidity during the growing season, and the highest percentages were recorded in winter and spring months especially in March.

In General, low temperature and high relative humidity due to increase in land snail population, therefore the caused damage to different cr0ps will also increase. These findings proved also that the increasing in damage appears obviously in month later than increased in population; February had highest population and March had the highest damage 


\section{REFERENCES}

AbdEl-Aal, S.M. (2001). Studies on certain land snails at Sharkia Governorate. M. Sc. Thesis, Fac. Agric. Zagazig. UNiv., 137pp.

Baker, G. H. (1988). The life history, population dynamics and polymorphism of Cernuella virgata ( Mollusca: Helicidae). Aust. J. Zool, 36, 497-512.

El- Deeb, H. I.; Wilson, M. and Eshra, E. H. (1999). Ecological studies on certain land snails infesting some economic crops at Behera Governorate, Egypt. Second Int. Conf. of Pest Control, Mansoura, Egypt, Sept., 1999; 19-27.

El-Okda, M.M.K. (1984). Land molluscs infestation and chemical control in ElIsmailia Governorate. J. Agric. Res. Rev., 62(1): 87-92.

Ismail, Sh. A. (1997). Ecology, biology and control of certain terrestrial snails infesting some vegetables and field crops in Sharkia Governorate. Ph.D. Thesis. Fac. Agric. Zagazig Univ.128 pp.

Ismail, Sh. A. (2004). Ecobiological studies on the brown garden snail, Eobania vermiculata (Muller) under laboratory and field conditions in Sharkia Governorate. Zagazig. J. Agric. Res., 31 (1): 293 - 305.

Gada Refaat (2004) Ecological and biological studies on some species of snails, Thesis , master of scince, Faculty of Agric., Moshtohor, Zagazig Univ.

Okka, M. A. (2003), Land snails Monacha contiana (Montagu) injurious to Egyptian Clover at Kafer El-Sheikh Governorate. J. Agric. Res. Tanta Univ., 29(2): 283-290.

Sebay, Z. S. Shetaia, Shahata A. A. Ismail and Samah M. AbdelKader,(2009),Survey, population dynamics and importance value of certain land snails species infesting different crops in sharkia Governorate, Egypt, Acadim., J. biolog. Sci., 1 (1):37-43(2009). 
Asran, A. A. et al.

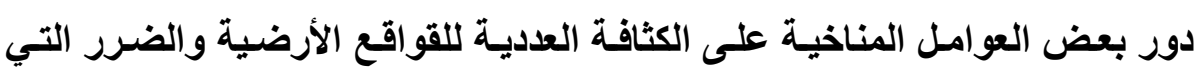

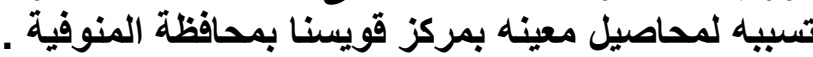

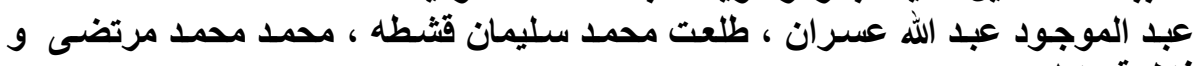
فاطمة كامل خضر المرد مركز البحوث الزراعيةـ معهد بحوث وقاية النباتات ـ الدقي- جمهورية مصر العربية

لقد نفذت هذه الدراسة تحت الظروف الحقلية لمركز قويسنا محافظة المنوفية خلال عامي توري

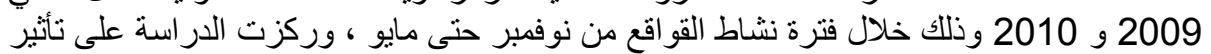

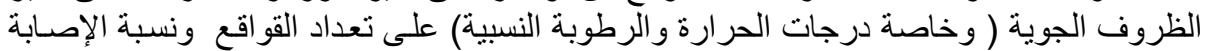

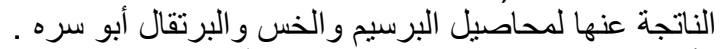
وأمكن تلخيص النتائج في النقاط التالية :- النيان

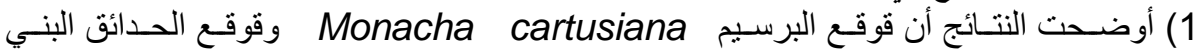

Eobania vermiculata

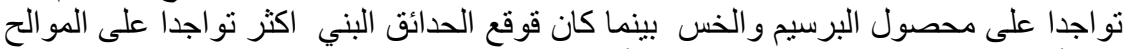

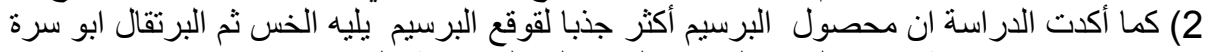

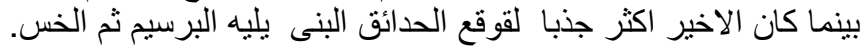

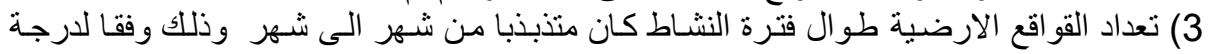

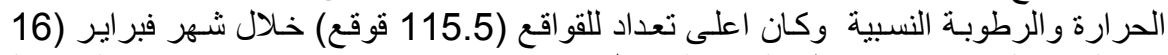

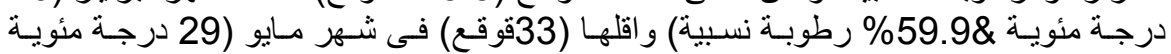

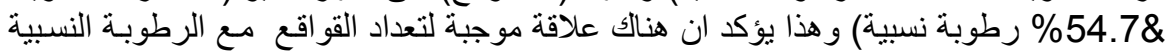
وسالبة مع درجة الحر ارة.

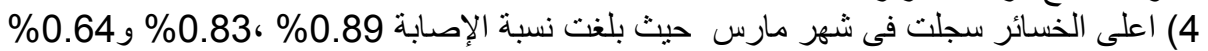

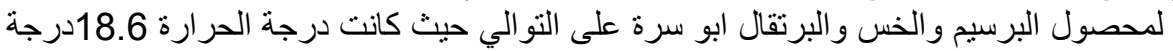

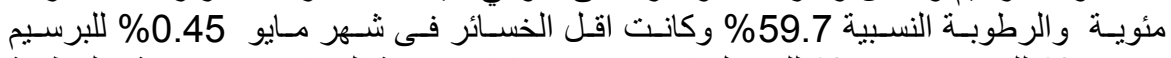

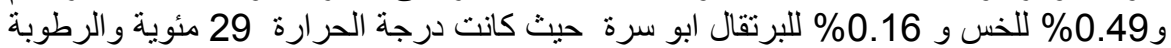

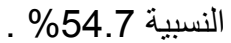
5) أكدت النتائج المتحصل عليها إن أكثر الظروف الجوية ملائمسة لزيادة تعداد القو اقع الأرضية

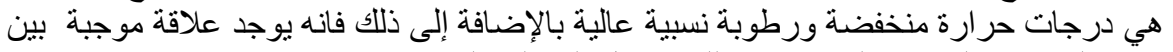
تعداد القواقع والخسائر الناتجة عنها للمحاصيل المختلفة الثلاثة.

كلية الزراعة - جامعة المنصورة مركز البحوث الزراعية

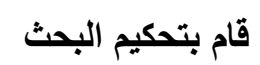

أ. أد / عمر عبد البحث الحميد نصار

أ.د / فاطمه شوقى المحروقى الصديد 
Table (1): Effect of temperature degree and relative humidity on the population density of land Snails infesting different field crops at Quessina district, Minufya governorate during two successive year 2009-2010

\begin{tabular}{|c|c|c|c|c|c|c|c|c|c|c|c|c|c|c|c|c|c|c|c|c|c|}
\hline \multirow{4}{*}{ Month } & \multirow{4}{*}{ Year } & \multirow{3}{*}{\multicolumn{2}{|c|}{$\begin{array}{c}\text { Average } \\
\text { of }\end{array}$}} & \multirow{2}{*}{\multicolumn{6}{|c|}{ Clover }} & \multirow{2}{*}{\multicolumn{6}{|c|}{ Lettuce }} & \multirow{2}{*}{\multicolumn{6}{|c|}{ Navel orange }} \\
\hline & & & & & & & & & & & & & & & & & & & & & \\
\hline & & & & \multirow[t]{2}{*}{\begin{tabular}{l|} 
Total \\
Colle.
\end{tabular}} & \multirow[t]{2}{*}{$\begin{array}{c}\text { Rate } \\
\text { to } \\
\text { may } \\
\end{array}$} & \multicolumn{2}{|c|}{$\begin{array}{c}\text { M. } \\
\text { cartusiana }\end{array}$} & \multicolumn{2}{|c|}{$\begin{array}{c}\text { E. } \\
\text { vermiculata }\end{array}$} & \multirow{2}{*}{$\begin{array}{l}\text { Total } \\
\text { Colle. }\end{array}$} & \multirow{2}{*}{$\begin{array}{c}\text { Rate } \\
\text { to } \\
\text { may }\end{array}$} & \multicolumn{2}{|c|}{$\begin{array}{c}M . \\
\text { cartusiana }\end{array}$} & \multicolumn{2}{|c|}{$\begin{array}{c}E . \\
\text { vermiculata }\end{array}$} & \multirow{2}{*}{$\begin{array}{l}\text { Total } \\
\text { Colle. }\end{array}$} & \multirow{2}{*}{$\begin{array}{c}\text { Rate } \\
\text { to } \\
\text { may }\end{array}$} & \multicolumn{2}{|c|}{$\begin{array}{c}\text { M. } \\
\text { cartusiana }\end{array}$} & \multicolumn{2}{|c|}{$\begin{array}{c}E . \\
\text { vemiculata }\end{array}$} \\
\hline & & C & R.h & & & No. & $\%$ & No & $\%$. & & & No. & $\% \%$ & No. & $\%$ & & & No & $\%$ & No. & $\%$ \\
\hline & 2009 & 21.4 & 62.3 & 44.3 & \multirow[t]{3}{*}{1.61} & 44 & 100 & 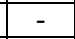 & - & 28.4 & \multirow[t]{3}{*}{1.34} & 28.4 & 100 & - & - & 28.4 & \multirow{3}{*}{-1.45} & 7.4 & 26 & 21 & 74 \\
\hline \multirow{2}{*}{ November } & 2010 & 16.5 & 57.5 & 62.7 & & 58 & 93.5 & 4 & 6.5 & 30.8 & & 26.2 & 85 & 4.6 & 15 & 21.2 & & 6.6 & 31.1 & 14.6 & 68.9 \\
\hline & mean & 19.0 & 59.9 & 53.5 & & 51 & 96.2 & 2 & 3.8 & 29.6 & & \begin{tabular}{|l|}
27.3 \\
\end{tabular} & 92.2 & 2.3 & 7.8 & 24.8 & & 7 & 28.5 & 17.8 & 71.5 \\
\hline & 2009 & 16.1 & 65.7 & 69 & \multirow[t]{3}{*}{2.15} & 65 & 94.2 & 4 & 5.8 & 33.5 & \multirow[t]{3}{*}{1.77} & 33.5 & 100 & - & - & 28 & \multirow[t]{3}{*}{1.82} & 10 & 35.7 & 18 & .364 \\
\hline \multirow{2}{*}{ December } & 2010 & 14.8 & 63.7 & 74 & & 67 & 91.8 & 6 & 8.2 & 37.3 & & 37.3 & 100 & - & - & 34 & & 10 & 29.4 & 24 & 70.6 \\
\hline & mean & 15.5 & 64.7 & 71.5 & & 66 & 93 & 5 & 7 & 35.4 & & \begin{tabular}{|l|}
35.4 \\
\end{tabular} & 100 & - & & 31 & & 10 & 32.5 & 21 & 67.5 \\
\hline & 2009 & 15.9 & 63.7 & 89 & 2.79 & 79 & 88.8 & 10 & 11.2 & 59 & 2.9 & 58 & 98.3 & 1 & 1.7 & 43 & 2.47 & 20 & 46.5 & 23 & 53.5 \\
\hline January & 2010 & 14.3 & 55.4 & 95 & & 83 & 87.4 & 12 & 12.6 & 57 & & 52 & 91.2 & 5 & 8.8 & 41 & & 16 & 39 & 25 & 61 \\
\hline & mean & 15.1 & 59.6 & 92 & & 81 & 88 & 11 & 12 & 58 & & 55 & 94.8 & 3 & 5.2 & 42 & & 18 & 42.9 & 24 & 57.1 \\
\hline & 2009 & 15.8 & 66.3 & 110 & 3.5 & 96 & 87.3 & 14 & 12.7 & 61.6 & 3.44 & 61.6 & 100 & - & - & 50 & 3.0 & 21 & 42 & 29 & 58 \\
\hline February & 2010 & 16.2 & 53.4 & 121 & & 105 & 86.8 & 16 & 13.2 & 76 & & \begin{tabular}{|l|}
76 \\
\end{tabular} & 100 & - & - & 52 & & 13 & 25 & 39 & 75 \\
\hline & mean & 16.0 & 59.9 & 115.5 & & 100.5 & 87 & 15 & 13 & 68.8 & & \begin{tabular}{|l|}
68.8 \\
\end{tabular} & 100 & - & - & 51 & & 17 & 33.3 & 34 & 66.7 \\
\hline & 2009 & 18.2 & 65.9 & 68.2 & 2.36 & 68 & 100 & - & - & 34.7 & 1.88 & 30.2 & 87 & 4.5 & 13 & 29 & 2.0 & 7 & 24.1 & 22 & 75.9 \\
\hline Marsh & 2010 & 19.0 & 53.4 & 88.2 & & 72 & 81.8 & 16 & 18.2 & 40.5 & & \begin{tabular}{|l|}
39 \\
\end{tabular} & 97.5 & 1.5 & 3.7 & 39 & & 17 & 43.6 & 22 & 56.4 \\
\hline & mean & 18.6 & 59.7 & 78.2 & & 70 & 89.7 & 8 & 10.3 & 37.6 & & \begin{tabular}{|l|}
34.6 \\
\end{tabular} & 92 & 3 & 8 & 34 & & 12 & 35.3 & 22 & 64.7 \\
\hline & 2009 & 21.4 & 59.2 & 59 & 1.79 & 54 & 91.5 & 5 & 8.5 & 32 & \begin{tabular}{|l|}
1.44 \\
\end{tabular} & 26.4 & 82.5 & 5.6 & 17.5 & 19.3 & $\begin{array}{ll}1.2 \\
\end{array}$ & 3.8 & 19.7 & 15.5 & 80.3 \\
\hline April & 2010 & 23.1 & 51.0 & 58 & & 50 & 87.7 & 7 & 12.3 & 25.6 & & \begin{tabular}{|l|}
21.6 \\
\end{tabular} & 84.3 & 4 & 15.7 & 21.5 & & 10.2 & 47.5 & 11.3 & 52.5 \\
\hline & mean & 22.3 & 55.1 & 58.5 & & 52 & 89.7 & 6 & 10.3 & 28.8 & & 24 & 83.4 & 4.8 & 16.6 & 20.4 & & 7 & 34.3 & 13.4 & 65.7 \\
\hline & 2009 & 31.7 & 56.0 & 30 & 1 & 30 & 100 & - & - & 19 & 1 & 18 & 94.8 & 1 & 5.2 & 15 & 1 & 7 & 46.7 & 8 & 53.3 \\
\hline May & 2010 & 26.2 & 53.4 & 36 & & 34 & 94.4 & 2 & 5.6 & 21 & & 20 & 95.2 & 1 & 4.8 & 19 & & 9 & 47.4 & 10 & 52.6 \\
\hline & mean & 29.0 & 54.7 & 33 & & 32 & 97 & 1 & 3 & 20 & & 17 & 95 & 1 & 5 & 17 & & 8 & 47 & 9 & 53 \\
\hline
\end{tabular}

